This is the author's manuscript for publication. The publisher-formatted version may be available through the publisher's web site or your institution's library.

\title{
Heterogeneous effects of warming and drought on selected wheat variety yields
}

Jesse Tack, Andrew Barkley, and Lawton Lanier Nalley

\section{How to cite this manuscript}

If you make reference to this version of the manuscript, use the following information:

Tack, J., Barkley, A., \& Nalley, L. L. (2014). Heterogeneous effects of warming and drought on selected wheat variety yields. Retrieved from http://krex.ksu.edu

\section{Published Version Information}

Citation: Tack, J., Barkley, A., \& Nalley, L. L. (2014). Heterogeneous effects of warming and drought on selected wheat variety yields. Climatic Change 125(3-4), 489-500.

Copyright: ㅇ Springer Science+Business Media Dordrecht 2014

Digital Object Identifier (DOI): doi:10.1007/s10584-014-1185-1

Publisher's Link: http://link.springer.com/article/10.1007/s10584-014-1185-1

This item was retrieved from the K-State Research Exchange (K-REx), the institutional repository of Kansas State University. K-REx is available at http://krex.ksu.edu 
Title: Heterogeneous Effects of Warming and Drought on Selected Wheat Variety Yields

Short Title: Warming and Drought Effects on Wheat Yield

Authors: Jesse Tack, Andrew Barkley, and Lawton Lanier Nalley

Corresponding Author: Jesse Tack: tack@agecon.msstate.edu; 662-325-7999 (phone); 662325-8777 (fax)

Abstract: Climate change is likely to significantly impact agricultural production in the Great Plains region of the Central United States. This study estimated the impact of changes in temperature and precipitation on wheat (triticum aestivum) variety yield distributions using the moment-based maximum entropy (MBME) model. This approach allows for quantification of potential weather impacts on the yield distribution, and allows these effects to vary across varieties. The unique data set matches wheat variety trial data for 1985 to 2011 with weather data from the exact trial site for 11 locations throughout Kansas. Ten widely-planted varieties with a range of biotic and abiotic characteristics were included for comparison. Weather scenarios were simulated for baseline, increased temperature (one-degree Celsius warming), decreased precipitation (tenth-percentile rainfall outcome), and a combination warming and drought scenario. Warming resulted in an 11 percent yield reduction, drought a 22 percent reduction, and warming and drought a cumulative 33 percent reduction. These effects vary across varieties. Alternative measures of yield risk (e.g. yield variance and coefficient of variation) were also constructed under each scenario and a similar pattern of heterogeneous impacts emerges. The key findings are that (i) exposure to warming and drought lead to mean yield reductions coupled with increased yield risk for all varieties, and (ii) newer (post 2005) seed varieties have a yield advantage over older varieties, however this advantage is reduced under warming and drought conditions.

Affiliations: Tack is Assistant Professor in the Department of Agricultural Economics at Mississippi State University, Starkville, Mississippi, USA; Barkley is University Distinguished Teaching Scholar and Professor in the Department of Agricultural Economics at Kansas State University, Manhattan, Kansas, USA; Nalley is Associate Professor in the Department of Agricultural Economics and Agribusiness at the University of Arkansas, Fayetteville, Arkansas, USA. 


\section{Introduction}

Current Intergovernmental Panel on Climate Change (IPCC) models project mean global surface temperature increases between $1.8^{\circ} \mathrm{C}$ and $4^{\circ} \mathrm{C}$ over the 21 st century at a rate of $0.2^{\circ} \mathrm{C}$ increase per decade (IPCC, 2007). As surface temperatures increase, the intensity and frequency of hightemperature events could increase substantially, thus reducing agricultural crop yields in many parts of the world. A large and growing literature has quantified these projected impacts (e.g. Schlenker and Roberts 2009; Mendelsohn et al. 1994; Adams et al. 1998, 1999). Climate change is likely to have a significant impact on agricultural production in the Great Plains region of the Central United States. Joyce et al. (2000) reported that changes in climate could result in crop shifts away from traditional crops such as wheat, an increase in invasive species of plants and animals, an increase in the need for irrigation, reductions in soil quality, increases in flooding and soil erosion, and pressure on rural economies.

Climate change is likely to have a major impact on global agricultural production, but the potential effects on crop yield and yield risk for wheat are not well understood (Lobell and Field 2007). Tubiello et al. (2002) projected that climate change will significantly affect rainfed wheat production in the Great Plains. They projected 10 to 50 percent decreases in hard winter wheat yields with higher variability in yields in the southern Great Plains region, thus increasing yield risk to producers. Ortiz et al. (2008) concluded that as weather patterns such as hotter temperatures, shorter growing seasons, and less rainfall occur, cultivar selection will become increasingly important to help mitigate yield risk. Semenov et al. (1996) highlighted the need to include climate variation when modeling wheat yields.

Although climate change is predicted to have a negative impact on future wheat yields in the Great Plains, genetic improvement via selective plant breeding is likely to offset at least some 
of the impact. In recent years, private companies and the public sector have made large investments in and improvements to wheat breeding programs (Battenfield et al. 2013). Measuring the ability of wheat breeders to offset biotic and abiotic stress and thus increase future wheat yields is important given the need to feed the large and increasing global population. Schmidt (1984) noted that increases in grain yield potential in performance trials from 1975 to 1984 in the Great Plains were minimal, and suggested that the rate of genetic gain was slowing or reaching a plateau. Graybosch and Peterson (2010) concluded that relative grain yields of Great Plains hard red winter wheat may have peaked in the early to mid-1990s, which is not surprising given the absence of both hybrid and genetically modified wheat cultivars. Recent work by Gourdji et al. (2013) and Schoppach and Sadok (2013) demonstrate the adverse effect of high temperatures on wheat yields.

This study provides an accurate and up-to-date estimate of the relative yield for each wheat variety holding constant location and weather. We provide evidence of a yield advantage for new varieties released since 2005. Furthermore, we simulate yield outcomes under warming and drought conditions and find that these new varieties provide some protection relative to older varieties. Our results rely on the use of field-trial data, which can be appropriately considered a natural-experiment setting for identifying weather effects. Another strength of our sampling design is that we observe yields for each variety, rather than relying on the use of more aggregate data for which identification of variety-specific effects is difficult if not impossible.

Plausible future climate change scenarios were used to estimate variety-specific yield distributions. This information is crucial to understanding how climate change could affect yield and revenue risk for wheat producers in Kansas and the Great Plains, as well as for the wheat seed industry. In 2012, Kansas was the number one wheat-producing state in the United States, 
and produced 10.2 million tons of wheat, or 16.8 percent of the United States total (Kansas 2013). In addition, insurance product designers require this information to offer products that meet the needs of producers.

Our findings provide initial estimates that can be used to construct a portfolio of wheat varieties to mitigate risk, which is important in mitigating the effects of climate change (Collier et al. 2009), and extends previous research of Barkley et al. (2010) and Nalley and Barkley (2010). Model results for wheat varieties provide wheat breeders initial information about breeding for heat tolerance (Pradhan et al. 2012). Wheat varieties grown in Kansas are described in detail by Watson (2013).

Recent work most closely related to this project includes Cabas et al. (2010), who examined the impact of climate and non-climatic factors on the mean and variance of maize (Zea mays L.), soybean (Glycine max (L.) Merr.), and winter wheat yields in Southwestern Ontario, Canada. Chen et al. (2004) also investigated the impact of climate on yield variability, following Dixon et al. (1994), who measured maize yield response models. Lobell and Asner (2003) presented trends in United States agricultural yields, and Lobell and Field (2007) examined changes in global production of major crops due to climate variables.

Prior research using the economic approach to the climate/crop relationship provides a solid foundation upon which to expand our knowledge of how weather and climate affect agricultural production in Kansas and the Great Plains (Black and Thompson 1978; Hansen 1991; Kaufmann and Snell 1997; Brown and Rosenberg 1999; Southworth et al. 2002; Weiss et al. 2003; Long et al. 2006; Ferrise et al. 2011). These studies estimated the impact of weather on crop yield distributions using aggregate-level data and model simulations. Kunkel et al. (2013) projected several plausible climate change scenarios for the Great Plains region, with projections 
that showed increased temperatures and extreme weather conditions, demonstrating the importance of furthering our understanding of the impact of weather on wheat yield distributions. The previous literature summarized here provides a reference point for our results. Our findings extend previous studies by using location-specific data for individual varietal yields.

\section{Materials and Methods}

This research measures the effect of weather outcomes on the distribution of wheat yields for specific varieties. Wheat variety yield distributions were estimated using the moment-based maximum entropy (MBME) model of Tack et al. (2012). ${ }^{1}$ This modeling approach quantifies potential impacts on the entire yield distribution, which allows for conclusions on a broad range of risk measures. For example, MBME results can include the effect of simulated weather shocks on the probability of a below-mean yield outcome, a measure that has been recently used to quantify exposure to downside risk (Tack and Ubilava 2013). Yield densities were estimated for ten popularly sown Kansas wheat varieties, allowing for the determination of heterogeneous mean and risk effects across different varieties. We focus on estimating differences in how yield distributions of wheat varieties in Kansas change under one degree $\mathrm{C}$ warming and drought (tenth percentile rainfall outcome) scenarios.

We condition this distribution on weather outcomes, which allows us to quantify climatic effects not only on mean yield, but on commonly-used measures of yield risk as well. The specific measures of yield risk that are considered here are variance, skewness, coefficient of variation, certainty equivalent, and the probability of a below-mean outcome. Urban et al. (2012) used the coefficient of variation to measure yield risk while Tack and Ubilava (2012) used the

\footnotetext{
1 The MBME approach has been previously used to model cotton and corn yields in Tack et al. (2012) and Tack and Ubilava (2013), respectively.
} 
probability of a below-mean outcome to measure downside risk. This latter measure allows one to focus the risk measure on the lower tail of the yield distribution.

The MBME model is best viewed as an extension of Antle's (1983, 2010) momentsbased approach. The key distinction is that moments-based models can measure the effects of conditioning variables on specific moments of the distribution (e.g. mean and variance), whereas the MBME model can measure these effects on the entire yield distribution. A detailed description of the modeling approach is provided in Section A1 of the Supplementary Material.

The wheat yield data span 1985 to 2011, drawn from Kansas Performance Tests for Winter Wheat Varieties (Kansas Performance 2013). All yield data are for dryland (nonirrigated, rainfed) Hard Red Winter Wheat (HRWW), with some observations (roughly ten percent) of Hard White Wheat (HWW). ${ }^{2}$ Yields are measured in kilograms per hectare, and the data include 6,680 observations across 245 varieties and 11 locations in Kansas. To account for changes in technology over time, we rescale all yields outcomes to 2011 levels as in Harri et al. (2011). ${ }^{3}$ Details for this procedure are provided in Section A2.1 of the Supplementary Material. Identifying the effects of weather on winter wheat yields is challenging due to the long growing season that begins in September and ends in June: wheat is exposed to warm weather in the fall, cold weather in the winter, and warm weather again in the spring. The impact of weather extremes on wheat yields can vary enormously across different months of the growing season. ${ }^{4}$ This is not the case with other row crops such as corn, soybeans, rice (Oryza sativa L.) and

\footnotetext{
${ }^{2}$ We focus on non-irrigated (dryland) wheat as it is more sensitive to weather fluctuations. The effect of climate change on irrigated wheat would likely be less damaging, but would require the use of additional irrigation due to evaporation.

${ }^{3}$ While the rescaling approach of Harri et al. (2011) represents the contemporary approach for normalizing yields over time in the yield modeling literature, and is currently used by the Risk Management Agency for calculating crop insurance premium rates for area yield products, it might not be appropriate if climate change interacts with changes in technology.

${ }^{4}$ As an example, exposure to extreme cold during the winter may not damage the wheat, but freeze damage can occur once the plant enters its growing stage in the early spring.
} 
cotton (Gossypium hirsutum L.), where the growing season is confined to the warm spring and summer months.

Many data-based studies of weather impacts on crop yields use average temperature over the entire growing season, or subsets thereof. This method can mask extreme temperatures that occur within a month or even during a fraction of a day. The approach of Schlenker and Roberts (2006, 2009) is used here to obtain a fine-scale weather data set that can be used to identify nonlinear weather effects. Specifically, a distribution of temperatures within each day is approximated using a sinusoidal curve between the observed minimum and maximum temperatures. This distribution captures the time-of-exposure to each one-degree Celsius (C) temperature interval. If utilized, the inclusion of a full set of daily one-degree interval temperature variables in the regression equations would limit available degrees of freedom as it would require an additional 256 variables. Therefore, the one-degree intervals were aggregated to form three-degree intervals. This does not limit our ability to identify the effects of extreme temperatures as these outcomes are infrequently observed and thus aggregated in practice. ${ }^{5}$ In addition, daily observations were summed to a monthly scale. The effect of precipitation was measured using a quadratic function of cumulative rainfall, following Roberts et al. (2013). As with the temperature data, cumulative daily rainfall was measured on a monthly scale. ${ }^{6}$ The monthly measures include September through May. ${ }^{7}$

The weather data are daily observations collected at the specific location of each variety trial, resulting in a location-specific match between variety yield and weather data. Most other statistical studies in the climate change literature rely on weather estimates over broad

\footnotetext{
${ }^{5}$ For example, Schlenker and Roberts (2009) include all temperatures above $39^{\circ} \mathrm{C}$ in a single interval.

${ }^{6}$ Further aggregation of both temperature and precipitation to seasonal (Fall, Winter, Spring) measures was tested, but $F$-tests suggested rejection of this restriction at standard significance levels $(p<0.00)$.

${ }^{7}$ Since harvest in Kansas typically occurs during June, the data do not include weather during the final part of the growing season, or during harvest.
} 
geographical areas, which can introduce substantial noise and hinder identification of weather effects. More information on the weather data can be found in Section A2.3 of the Supplementary Material.

Our main focus is to measure the extent to which warming and drought conditions perturb wheat yield distributions for select varieties. Although we estimate the MBME model using all available varieties, we focus the empirical findings on ten varieties that were selected based on high adoption rates and diverse characteristics. Five of the included varieties were released before 2005: variety-2137, Jagalene, TAM 111, Overley, and TAM 112. Varieties released since 2006 included: Fuller, Duster, Armour, Everest, and T158. Our rationale for focusing on these varieties is provided in Section A2.2 of the Supplementary Material.

\section{Results and Discussion}

The first component of the MBME model uses regression analysis to condition the first three moments of the yield distribution on monthly precipitation and temperature outcomes. We find that the conditioning variables explain 78 percent of the variation in yields (Supplementary Table A2), a strong indication of model performance as the analysis is for plot-level yields. This explanatory capability also extends to the higher order moment equations. Further analysis of the moments model indicates that controlling for location and variety fixed effects are important modeling components, and that monthly temperature and precipitation outcomes throughout the long September-May growing season are important drivers of wheat yields (Supplementary Table A3). More details on the estimation procedure and model selection results are discussed in Section A3 of the Supplementary Material. 
The MBME model allows us to generate yield distributions conditioned on both climate and variety. We focus on four simulated climate scenarios for each variety: (1) a baseline temperature and precipitation scenario that conditions yields on the sample average of the weather outcomes; (2) a warming scenario in which precipitation is held at the same value as in (1) but temperatures are increased by one degree C; (3) a drought scenario in which temperatures are held at the same values as in (1), but precipitation is held at a value corresponding to the tenth percentile of its historical distribution of outcomes; and (4) a final scenario that combines the warming and drought scenarios. The estimated densities for the 40 variety-climate combinations are reported in Figures A13-A18 of the Supplementary Material. The general pattern of results suggests that both warming and drought shift the yield distribution closer to zero.

While these figures provide a qualitative analysis of the weather effects, it is difficult to infer the implied effects on the mean and risk of crop yields. Using the estimated densities, the mean, variance, coefficient of variation, certainty equivalents, and skewness were calculated for each variety-climate combination. ${ }^{8}$ These findings are reported in Table 1 , where mean yields under the baseline scenario exhibit heterogeneity across varieties. Newer varieties had approximately a 150 kilogram per hectare $\left(\mathrm{kg} \mathrm{ha}^{-1}\right)$ advantage relative to the older varieties. This provides some evidence that Kansas wheat yields increased since 2005, a result that differs from that of Graybosch and Peterson (2010). The alternative climate scenarios had a large impact on mean yields, with warming resulting in an 11 percent reduction, drought a 22 percent reduction, and the combination of the two a 33 percent reduction. While the effects are fairly homogeneous, there are some differences across varieties.

\footnotetext{
${ }^{8}$ The literature on agricultural production and risk management has long recognized the importance of the shape of the yield distribution for on-farm decision making (Chavas and Holt 1996; Moschini and Hennessy 2001; DiFalco and Chavas 2006, 2009; Antle 1983, 2010; and Du et al. (2012).
} 
Heterogeneity in variance exists across varieties under all four climate scenarios. Under the baseline scenario, variances ranged from 0.54 to 1.88 , with a 0.08 unit variance increase associated with the newer varieties, on average. The effects of warming and drought were heterogeneous as well, with variances increasing under warming and drought in isolation of each other, but sometimes decreasing in combination.

The variance in isolation can be a misleading measure of risk, as wheat producers are often interested in the tradeoff between mean and variances effects. Thus, we report coefficients of variation (CV) in column three of Table 1. This statistic is defined as the ratio of the standard deviation of yield over mean yield, providing a normalized (unitless) measure of risk. Heterogeneity exists across varieties, as the CVs ranged from 0.155 to 0.310 , and are essentially equivalent, on average, across the older and newer varieties ( 0.255 compared to 0.254 for the older varieties). The CVs increased under the warming, drought, and combined warming/drought scenarios. Our finding, coupled with large reductions in mean yields, provides further evidence of the detrimental effects of climate change.

While the CV provides a normalized measure of dispersion, it does not fully capture the combined effects on the mean and variance of wheat yields. A certainty equivalent (CE) measure of yield is the level of yield a producer would be willing to accept in return for the full elimination of yield variance. For a risk averse farmer (as is typically assumed in the literature) the certainty equivalent is less than mean yield because the farmer has a preference for a reduction in risk (i.e. yield variance). In this way, the CE depends on both risk exposure and risk preferences. CEs are calculated under a negative exponential utility function with the absolute risk aversion coefficient set equal to 0.15 , in which case $C E=m-.075 v$ where $m$ and $v$ are the mean and variance of yield. For example, in the first row in Table 1 the CE of 4.31 megagrams 
per hectare $\left(\mathrm{Mg} \mathrm{ha}^{-1}=1000 \mathrm{~kg} \mathrm{ha}^{-1}\right)$ implies that the producer is exactly indifferent between a certain (zero variance) yield of $4.31 \mathrm{Mg} \mathrm{ha}^{-1}$ and the distribution of yield outcomes with a mean of $4.40 \mathrm{Mg} \mathrm{ha}^{-1}$ and a variance of 1.15 . The CEs for the baseline scenario ranged from 4.25 to 4.79, with the average CEs for the newer varieties being approximately $146 \mathrm{~kg} \mathrm{ha}^{-1}$ higher than those for the older varieties. Thus, the newer varieties increase in mean yields while holding the coefficient of variation equivalent has been a positive technological development. Under the warming, drought, and combined scenarios, this difference was reduced to 135, 124, and $113 \mathrm{~kg}$ $\mathrm{ha}^{-1}$. This implies that warming and drought reduce the (absolute) yield advantage held by newer varieties. However, the yield advantage remained large across all scenarios. All three climate scenarios reduced the CEs for all varieties, with the CEs under the combined warming and drought scenario being the lowest.

The final column reports the skewness of the estimated variety-regime density. Under the baseline scenario, yields were positively skewed for all but one variety. Patterns of skewness across alternative variety-regime combinations were nuanced, as there is substantial heterogeneity across varieties within each weather scenario and across scenarios within each variety.

While skewness provides some insight into the probabilities of low-frequency yield outcomes, it is more insightful to construct these probabilities directly. Furthermore, it is possible to anchor these probabilities to the mean yield outcome under the baseline scenario so that they are directly comparable across the warming, drought, and combined warming/drought scenarios. Thus, downside risk was defined as the probability of an outcome below mean baseline yield. Estimates for each of the variety-climate combinations were constructed for downside risk. These probabilities are reported in the second column (probability of a yield outcome below 
mean) in Table 2. The probabilities for the baseline scenario were omitted, since they are necessarily 50 percent by construction. For each subsequent weather regime, we integrate the variety-climate specific density over the interval $\left[0, \mu_{i, r=1}\right]$, where $\mu_{i, r=1}$ is the mean yield for variety $i$ under the baseline weather scenario. By anchoring this probability, we capture downside risk in a meaningful way by measuring how far the distribution shifts to the left of the mean baseline yield. For example, under the warming scenario the probability increased roughly 14 percentage points to 64 percent. This implies that warming shifts the distribution left so that approximately 64 percent of all possible yield outcomes are below the baseline mean. The effect of drought is even more severe, with roughly 77 percent of all outcomes located below the mean. The combined effects of warming and drought are striking, with nearly all outcomes located below the baseline yield. Importantly, these probabilities are heterogeneous across varieties with the range of outcomes being 63.3 to 67.4 percent for the warming scenario, 74.7 to 81.6 for the drought scenario, and 85.4 to 96.0 percent for the combined scenario.

Given our defined climate scenarios, we can evaluate the difference in drought effects under baseline and warming scenarios. The drought scenario results show that drought increased the probability of a below-baseline-mean outcome by approximately 27 percent. Conversely, under the combined scenario, the increase is roughly 39 percentage points. This implies that droughts will have an increasingly negative effect when warming materializes as producer exposure to downside risk increases by approximately 12 percentage points.

The remaining columns of Table 2 provide alternative measures of downside risk. The original threshold for a "bad" yield outcome was the mean of the baseline distribution; three additional outcomes provide reductions to this threshold as a proportion of the mean. As these columns incrementally reduce the threshold from 90 to 70 to 50 percent of the mean, the measure 
of downside risk becomes increasingly focused on the lower tail of the distribution. Overall, we see that same pattern of results across these alternatives compared to the original threshold. The final column shows that what was once a rare outcome under the baseline scenario (less than five percent across all varieties) became increasingly frequent under the warming and drought scenarios, essentially becoming a fairly common (20 to 30 percent) outcome under the combined effects of warming and drought.

\section{Conclusions}

Given the current Intergovernmental Panel on Climate Change (IPCC) models which project mean global surface temperature increases between $1.8^{\circ} \mathrm{C}$ and $4^{\circ} \mathrm{C}$ over the 21 st century, the intensity and frequency of high-temperature events could increase substantially (IPCC, 2007). This would in turn have a global effect on the mean and variance of wheat yields. This research provides an initial step toward the identification and construction of optimal wheat yield variety portfolios for potentially mitigating these climatic changes. Wheat variety yield distributions for ten widely-planted Kansas varieties were estimated using location-specific weather data for 11 locations throughout Kansas. The yield distributions were estimated for four plausible future climate scenarios: (1) baseline, (2) one-degree C temperature warming, (3) drought conditions (tenth percentile rainfall outcome), and (4) a combination of warming and drought.

Results suggest that climate has heterogeneous effects on wheat yield distributions across varieties. Mean yields are found to decline under both warming and drought, and coefficients of variation increased under warming and drought separately and in combination. This parallels the finding in Urban et al. (2012) who found evidence of an increase in the coefficient of variation for corn yields under climate change and Gourdji et al. (2013), who found decreases in yield 
advantages of new wheat varieties globally. These coefficients represent a simple and straightforward way to quantify risk. We consider others - including certainty equivalents and measures of downside risk - and find that the pattern of results is robust to these alternatives. Our key finding is that exposure to warming and drought lead to mean yield reductions, coupled with increased yield risk. Importantly, these impacts differ across varieties. Newer (post 2005) seed varieties have higher mean yields than older varieties, but adverse weather outcomes reduce the yield advantage of newer varieties.

Certainty equivalents were calculated and found to be much larger for the newer varieties under baseline climate conditions. This advantage was maintained under the warming and drought scenarios, however the size of the advantage declined under both scenarios. This implies that warming and drought reduce the (absolute) yield advantage held by newer varieties. Exposure to downside risk, measured as the probability of a below-mean outcome, was estimated as well. We find that drought increased this probability by 27 percentage points, and the combined warming and drought scenario resulted in an increase of 40 percentage points. Therefore, the occurrence of drought conditions are likely to have an increasingly negative effect on yields and yield risk under a warmer climate.

The estimation of separate and combined effects of temperature and precipitation on wheat variety yields will continue to provide useful information as climate changes occur. Continued observation and estimation with statistical models such as the one presented here, between the interaction of increasingly variable weather conditions and wheat yields, will provide for refinement and enhancement of this modeling approach. The results provide plant breeders, agricultural policy makers, and private enterprises with important direction for sustaining wheat production in an ever-changing climatic world. 


\section{Acknowledgements}

Special thanks to: Robert Bowden, Forrest Chumley, Arlin Feyerherm, Allan Fritz, Jane Lingenfelser, Zara Niederman, and James Shroyer. 
Table 1. Wheat Variety Yield Mean and Risk Effects under Climate Scenarios

\begin{tabular}{|c|c|c|c|c|c|}
\hline Variety & mean $\left(1000 \mathrm{~kg} \mathrm{ha}^{-1}\right)$ & variance & coeff variation & cert equivalent & skewness \\
\hline \multicolumn{6}{|c|}{ Baseline Temperature and Precipitation (Scenario 1) } \\
\hline 2137 & 4.40 & 1.15 & 0.243 & 4.31 & 0.070 \\
\hline Jagalene & 4.40 & 1.66 & 0.293 & 4.28 & 0.058 \\
\hline Overley & 4.47 & 1.65 & 0.288 & 4.35 & -0.168 \\
\hline TAM 111 & 4.37 & 1.60 & 0.289 & 4.25 & 0.089 \\
\hline TAM 112 & 4.73 & 0.54 & 0.155 & 4.69 & 0.043 \\
\hline Armour & 4.46 & 1.73 & 0.294 & 4.33 & 0.094 \\
\hline Duster & 4.43 & 1.88 & 0.310 & 4.29 & 0.103 \\
\hline Fuller & 4.86 & 0.93 & 0.198 & 4.79 & 0.063 \\
\hline T158 & 4.69 & 1.09 & 0.223 & 4.61 & 0.069 \\
\hline Everest & 4.69 & 1.39 & 0.251 & 4.59 & 0.079 \\
\hline \multicolumn{6}{|c|}{ One Degree Warming with Baseline Precipitation (Scenario 2) } \\
\hline 2137 & $3.89(-11.5 \%)$ & $1.67(46.4 \%)$ & $0.333(36.7 \%)$ & 3.77 & 0.110 \\
\hline Jagalene & 3.89 (-11.4\%) & 2.18 (31.7\%) & 0.379 (29.6\%) & 3.73 & 0.163 \\
\hline Overley & 3.96 (-11.3\%) & $2.26(36.7 \%)$ & 0.379 (31.8\%) & 3.80 & 0.164 \\
\hline TAM 111 & 3.86 (-11.5\%) & 2.09 (30.9\%) & 0.374 (29.4\%) & 3.71 & 0.155 \\
\hline TAM 112 & $4.22(-10.6 \%)$ & 1.43 (166\%) & 0.283 (82.9\%) & 4.12 & 0.083 \\
\hline Armour & 3.95 (-11.3\%) & 2.31 (33.9\%) & 0.384 (30.5\%) & 3.78 & 0.170 \\
\hline Duster & $3.91(-11.4 \%)$ & 2.42 (28.9\%) & 0.397 (28.2\%) & 3.74 & 0.188 \\
\hline Fuller & 4.35 (-10.4\%) & 1.94 (110\%) & 0.320 (61.8\%) & 4.21 & 0.072 \\
\hline T158 & $4.18(-10.7 \%)$ & 1.93 (77.2\%) & 0.332 (49.2\%) & 4.04 & 0.116 \\
\hline Everest & 4.18 (-10.7\%) & 2.22 (60.1\%) & 0.356 (41.8\%) & 4.02 & 0.140 \\
\hline \multicolumn{6}{|c|}{ Baseline Temperature with Drought (Scenario 3) } \\
\hline 2137 & $3.41(-22.2 \%)$ & $1.24(8.84 \%)$ & $0.326(34.2 \%)$ & 3.32 & -0.160 \\
\hline Jagalene & $3.42(-22.2 \%)$ & 1.74 (4.93\%) & 0.385 (31.7\%) & 3.29 & -0.022 \\
\hline Overley & 3.49 (-21.9\%) & $1.86(13.0 \%)$ & 0.391 (36.1\%) & 3.35 & -0.153 \\
\hline TAM 111 & $3.39(-22.4 \%)$ & 1.62 (2.01\%) & $0.376(30.1 \%)$ & 3.27 & 0.148 \\
\hline TAM 112 & $3.74(-20.7 \%)$ & $1.29(141 . \%)$ & $0.304(96.1 \%)$ & 3.65 & -0.632 \\
\hline Armour & 3.48 (-21.9\%) & 1.92 (11.6\%) & 0.398 (35.4\%) & 3.34 & 0.181 \\
\hline Duster & 3.44 (-22.1\%) & 2.00 (6.50\%) & 0.410 (32.5\%) & 3.30 & 0.199 \\
\hline Fuller & 3.87 (-20.1\%) & 1.91 (106\%) & 0.356 (80.2\%) & 3.73 & -0.377 \\
\hline T158 & $3.71(-20.8 \%)$ & 1.75 (60.7\%) & 0.356 (60.2\%) & 3.58 & -0.277 \\
\hline Everest & $3.71(-20.8 \%)$ & $2.04(47.2 \%)$ & 0.385 (53.3\%) & 3.56 & -0.006 \\
\hline \multicolumn{6}{|c|}{ One Degree Warming with Drought (Scenario 4) } \\
\hline 2137 & $2.91(-33.7 \%)$ & $0.79(-30.4 \%)$ & 0.306 (25.9\%) & 2.85 & -0.241 \\
\hline Jagalene & $2.91(-33.7 \%)$ & $1.28(-22.5 \%)$ & 0.389 (32.8\%) & 2.82 & 0.155 \\
\hline Overley & $2.98(-33.2 \%)$ & $1.48(-10.3 \%)$ & $0.408(41.8 \%)$ & 2.87 & 0.185 \\
\hline TAM 111 & $2.88(-34.0 \%)$ & $1.13(-28.8 \%)$ & 0.369 (27.8\%) & 2.80 & 0.129 \\
\hline TAM 112 & 3.24 (-31.4\%) & $1.18(120 \%)$ & $0.335(116 \%)$ & 3.15 & -0.211 \\
\hline Armour & $2.97(-33.3 \%)$ & $1.51(-12.2 \%)$ & 0.413 (40.4\%) & 2.86 & 0.194 \\
\hline Duster & $2.93(-33.6 \%)$ & $1.54(-17.5 \%)$ & 0.423 (36.7\%) & 2.82 & 0.209 \\
\hline Fuller & 3.37 (-30.5\%) & $1.92(108 \%)$ & $0.411(107 \%)$ & 3.23 & 0.199 \\
\hline$T 158$ & $3.20(-31.6 \%)$ & 1.59 (46.2\%) & 0.394 (76.9\%) & 3.09 & 0.169 \\
\hline Everest & $3.20(-31.6 \%)$ & $1.86(34.4 \%)$ & $0.426(69.7 \%)$ & 3.07 & 0.220 \\
\hline
\end{tabular}

Notes: Table reports the means, variances, coefficients of variation, certainty equivalents, and skewness for a selection of ten varieties across the baseline and climate change scenarios. Certainty equivalents (CE) are calculated under a negative exponential utility function with the absolute risk aversion coefficient set equal to 0.15 , in which case $C E=m-.075 \mathrm{v}$ where $m$ and $v$ are the mean and variance of yield. For the mean, variance, and coefficient of variation columns, the values in parentheses report the percentage change in the value relative to its baseline counterpart. 
Table 2. Wheat Variety Yield Downside Risk Effects under Climate Scenarios

\begin{tabular}{|c|c|c|c|c|}
\hline \multirow[b]{2}{*}{ Variety } & \multicolumn{4}{|c|}{ Probability of a yield outcome below.... } \\
\hline & mean & 0.9mean & 0.7mean & 0.5mean \\
\hline \multicolumn{5}{|c|}{ Baseline Temperature and Precipitation (Scenario 1) } \\
\hline 2137 & -- & $34.1 \%$ & $11.1 \%$ & $1.82 \%$ \\
\hline Jagalene & -- & $36.5 \%$ & $15.6 \%$ & $4.24 \%$ \\
\hline Overley & -- & $34.6 \%$ & $15.2 \%$ & $4.79 \%$ \\
\hline TAM 111 & -- & $37.4 \%$ & $14.7 \%$ & $4.03 \%$ \\
\hline TAM 112 & -- & $25.8 \%$ & $2.42 \%$ & $0.04 \%$ \\
\hline Armour & -- & $36.7 \%$ & $14.9 \%$ & $4.35 \%$ \\
\hline Duster & -- & $38.3 \%$ & $16.7 \%$ & $4.95 \%$ \\
\hline Fuller & -- & $30.1 \%$ & $6.29 \%$ & $0.52 \%$ \\
\hline$T 158$ & -- & $32.1 \%$ & $8.97 \%$ & $1.10 \%$ \\
\hline Everest & -- & $34.1 \%$ & $11.7 \%$ & $2.13 \%$ \\
\hline \multicolumn{5}{|c|}{ One Degree Warming with Baseline Precipitation (Scenario 2) } \\
\hline 2137 & $65.7 \%$ (15.1pp) & $52.4 \%$ (18.2pp) & 27.4\% (16.3pp) & $9.46 \%$ (7.64pp) \\
\hline Jagalene & $63.9 \%$ (13.7pp) & $52.3 \%$ (15.7pp) & $30.2 \%$ (14.6pp) & $12.6 \%$ (8.44pp) \\
\hline Overley & $63.3 \%$ (15.2pp) & $51.8 \%$ (17.1pp) & $30.1 \%$ (14.8pp) & $12.8 \%$ (8.02pp) \\
\hline TAM 111 & 63.7\% (13.8pp) & $53.1 \%$ (15.7pp) & $29.3 \%$ (14.5pp) & $12.5 \%$ (8.51pp) \\
\hline TAM 112 & 67.4\% (15.9pp) & $51.4 \%$ (25.6pp) & $22.1 \%$ (19.7pp) & $5.66 \%$ (5.61pp) \\
\hline Armour & 63.4\% (13.2pp) & $52.0 \%$ (15.3pp) & $29.4 \%$ (14.4pp) & $13.2 \%$ (8.88pp) \\
\hline Duster & $64.0 \%$ (12.7pp) & $53.0 \%$ (14.7pp) & $30.8 \%$ (14.1pp) & $13.6 \%$ (8.72pp) \\
\hline Fuller & $64.2 \%$ (14.1pp) & $50.3 \%$ (20.2pp) & $24.9 \%$ (18.6pp) & $8.57 \%$ (8.04pp) \\
\hline$T 158$ & 64.8\% (14.1pp) & 51.0\% (18.8pp) & 26.5\% (17.6pp) & $9.18 \%$ (8.08pp) \\
\hline Everest & 64.0\% (13.3pp) & 51.1\% (16.9pp) & 28.1\% (16.4pp) & $10.8 \%$ (8.72pp) \\
\hline \multicolumn{5}{|c|}{ Baseline Temperature with Drought (Scenario 3) } \\
\hline 2137 & 80.8\% (30.2pp) & $67.2 \%$ (33.1pp) & $37.6 \%$ (26.4pp) & $14.1 \%$ (12.2pp) \\
\hline Jagalene & $76.4 \%$ (26.2pp) & $64.7 \%$ (28.2pp) & $40.0 \%$ (24.3pp) & $18.3 \%$ (14.0pp) \\
\hline Overley & $74.7 \%$ (26.6pp) & $62.7 \%$ (28.0pp) & $38.7 \%$ (23.5pp) & $18.8 \%$ (14.0pp) \\
\hline TAM 111 & $77.5 \%$ (27.6pp) & $67.3 \%$ (29.9pp) & $40.2 \%$ (25.5pp) & $17.8 \%$ (13.8pp) \\
\hline TAM 112 & $81.6 \%$ (30.1pp) & $64.3 \%$ (38.4pp) & $30.3 \%$ (27.9pp) & $11.3 \%$ (11.3pp) \\
\hline Armour & $75.9 \%$ (25.7pp) & $65.1 \%$ (28.4pp) & $40.1 \%(25.2 \mathrm{pp})$ & $19.2 \%$ (14.9pp) \\
\hline Duster & $76.3 \%$ (24.9pp) & $65.8 \%$ (27.5pp) & $41.4 \%$ (24.7pp) & $19.5 \%$ (14.5pp) \\
\hline Fuller & $74.6 \%$ (24.6pp) & $60.4 \%(30.2 \mathrm{pp})$ & $33.6 \%$ (27.3pp) & $15.5 \%$ (15.0pp) \\
\hline$T 158$ & $76.2 \%$ (25.5pp) & $62.1 \%$ (29.9pp) & $35.6 \%$ (26.6pp) & $15.6 \%$ (14.5pp) \\
\hline Everest & $74.9 \%$ (24.2pp) & $62.7 \%$ (28.5pp) & $38.5 \%$ (26.7pp) & $17.6 \%$ (15.5pp) \\
\hline \multicolumn{5}{|c|}{ One Degree Warming with Drought (Scenario 4) } \\
\hline 2137 & $96.0 \%$ (45.5pp) & $88.2 \%(54.1 \mathrm{pp})$ & $56.5 \%(45.4 p p)$ & 20.7\% (18.9pp) \\
\hline Jagalene & $90.1 \%$ (39.8pp) & $81.8 \%$ (45.2pp) & $57.1 \%(41.4 p p)$ & $27.0 \%$ (22.8pp) \\
\hline Overley & $88.2 \%$ (40.1pp) & $79.7 \%$ (45.0pp) & $56.2 \%$ (41.0pp) & $28.2 \%(23.4 p p)$ \\
\hline TAM 111 & 91.2\% (41.3pp) & $84.0 \%$ (46.6pp) & $56.7 \%$ (42.0pp) & $26.5 \%$ (22.5pp) \\
\hline TAM 112 & $92.4 \%$ (40.9pp) & 82.1\% (56.3pp) & $50.2 \%(47.7 p p)$ & $20.3 \%$ (20.3pp) \\
\hline Armour & 88.0\% (37.8pp) & 79.7\% (43.0pp) & $54.9 \%$ (39.9pp) & $28.7 \%$ (24.3pp) \\
\hline Duster & 88.3\% (37.0pp) & $80.2 \%$ (41.9pp) & $56.1 \%$ (39.4pp) & $28.7 \%$ (23.7pp) \\
\hline Fuller & 85.4\% (35.3pp) & $76.1 \%$ (46.0pp) & $51.8 \%$ (45.5pp) & $26.2 \%$ (25.6pp) \\
\hline$T 158$ & 87.8\% (37.1pp) & $78.4 \%$ (46.3pp) & $53.7 \%$ (44.8pp) & $25.6 \%$ (24.5pp) \\
\hline Everest & $85.9 \%$ (35.2pp) & $76.8 \%$ (42.6pp) & $53.8 \%$ (42.1pp) & $27.6 \%$ (25.5pp) \\
\hline
\end{tabular}

